\title{
Mapping Permanent Magnets with High Angular Accuracy
}

\author{
Sasa Spasic ${ }^{1}$, Marjan Blagojevic ${ }^{2}$, Nenad Markovic $^{2}$, Zarko Mitrovic $^{2}$, Lazar Popovic $^{2}$ and R.S. Popovic ${ }^{1}$ \\ ${ }^{1}$ SENIS AG, Neuhofstrasse 5a, 6340 Baar, Switzerland \\ ${ }^{2}$ SENTRONIS AD, Aleksandra Medvedeva 14, 18000 Nis, Serbia \\ info@sentronis.rs
}

\begin{abstract}
:
Modern magnetic position and angle sensors consist of a combination of a permanent magnet and a magnetic field sensor. The magnetic field of the permanent magnets used in position sensors must be mapped with high accuracy. The sensitivity vectors of the mapper's 3 -axis probe are subject to angular tolerances of a few degrees. This paper describes a method of calibration of the probe within the magnetic field mapper, which reduces the angular errors to under 0.1 degree. During the calibration, the integrated 3-axis Hall probe is placed in the Calibration Cube with the reference magnet and output voltages of the probe are read. This allows for determination of all 9 components of the probe's sensitivity tensor. Later, while mapping a magnet, the actual magnetic field vectors are calculated by multiplying the inverse sensitivity matrix by the vector of the probe's output voltages.
\end{abstract}

Key words: Magnetic Field Mapper, 3-axis Hall Probes, Hall Probes, Permanent Magnets, Position Sensors.

\section{Introduction}

Modern magnetic translational or angular position sensors consist of a combination of a permanent magnet and a 1-, 2-, or 3-axis magnetic field sensor. The position of the moving part is retrieved from the magnetic field vector of the magnet "seen" by the magnetic sensor. Since imperfections of the magnet cause errors of the measured position [1], the distribution of the magnetic field of the magnets has to be checked. These imperfections are caused either by magnet material or by the magnetizing process and can produce a magnetization angle of few degrees [2].

Often it is needed to measure all three components of the magnetic field vector at many points in space and to build a map of the magnetic field.

Instruments suitable for such measurements are known as magnetic field mappers (Fig. 1).

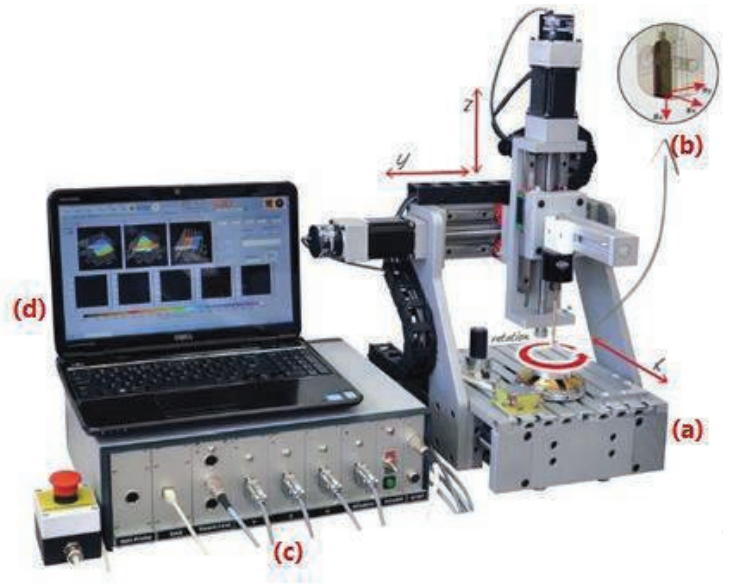

Fig. 1. The SENIS Magnetic Field Mapper MMS$1 A-R S$ consists of four main parts: (a) the mechanical part (the moving platform with three linear modules and the rotary stage); (b) the Hall Probe mounted on the Touch sensor; (c) the electronic box with digital signal processing, motor drives, encoder control, and power supply; (d) and a $P C$ and Software for measured data acquisition, visualization, and analysis

A good magnetic field mapper should meet the following requirements: (a) High magnetic and spatial resolution of the magnetic measurement (Fig. 2); (b) Measurement of all three components of a magnetic field at the same spot; (c) Good mechanics - accurate positioning of the probe, high speed, high positioning resolution, etc.; (d) High conformity of the 
sensitivity vectors of the probe with the coordinate system of the mapper.

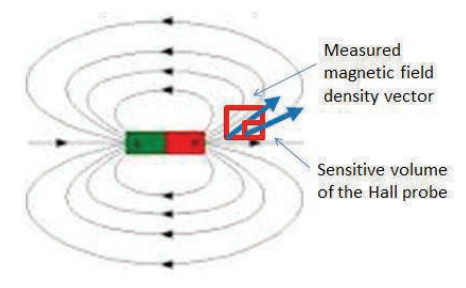

Fig. 2. Measuring of high inhomogeneous magnetic fields requires small and compact sensitive volume of the Hall probe and measuring all three components of a magnetic field simultaneously at the same spot. If the magnetic field density vector is measured with a probe that has a large sensitive volume as shown above, the measurement result will be the average of all magnetic fields lines seen by the probe's sensitive volume in this area. If a probe with a small and compact sensitive volume is used, a slightly different magnetic field density vector will be measured. In a non-homogeneous magnetic field, not only the amplitude, but also the orientation of the magnetic flux density vector is position-dependent; and therefore, all three components of the magnetic field should be measured at the same spot.

The requirements (a)-(c) are met by a commercially available magnetic field mapper [3] or by integrating a 3-axis Teslameter [4] in a coordinate measurement machine [5]. But the solution for the requirement (d) has been not found yet. The problem is the fact that, even in a CMOS integrated 3-axis Hall probe [6], the angular tolerances of the 3 sensitivity vectors could be larger than $1^{\circ}$; and assembling a small probe in a mapper is subject to additional angular tolerances [7].

The extraordinary feature of the Magnetic Field Mapper MMS-1A-RS is that it is equipped with a touch-sensor and a ruby ball stylus, which transforms the Mapper into a simple, easy-touse Coordinate Measuring Machine - CMM (Fig. 3) [3]. A touch-stylus, mounted on the console of the moving platform, allows for precise calibration of the position of the probe and can be used for the dimensional measurement of the tested magnets.
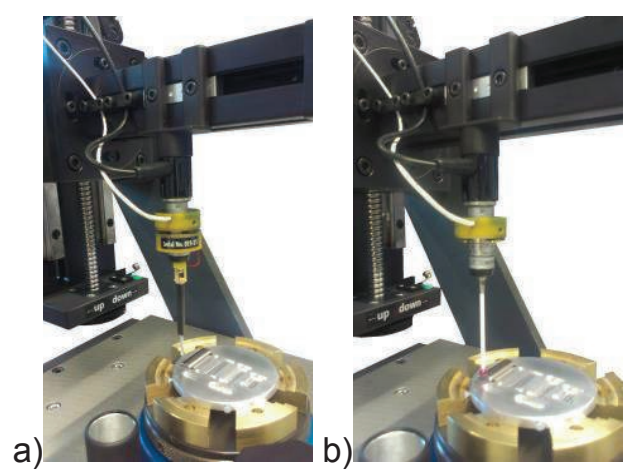

Fig. 3. a) The Hall probe mounted on a touch-trigger sensor of the magnetic field mapper; b) The touch- stylus mounted on a touch-trigger sensor of the magnetic field mapper (for the simple CMM functionality);

\section{Sensitivity Tensor of the Probe}

The output voltages of a three-axis Hall probe are given by the equation

$$
\left[\begin{array}{l}
V h_{x} \\
V h_{y} \\
V h_{z}
\end{array}\right]=\left[\begin{array}{ccc}
S x x & S x y & S x z \\
S y x & S y y & S y z \\
S z x & S z y & S z z
\end{array}\right] \bullet\left[\begin{array}{c}
B_{x} \\
B_{y} \\
B_{z}
\end{array}\right]
$$

where $\vec{B}$ denotes "the Hall voltage vector" (the three output voltages of the three-axis Hall probe); [S] is the probe's sensitivity tensor, whose 9 components are the components of the three sensitivity vectors of the Hall probe; and $\vec{V} h$ is the magnetic flux density vector - all in the common coordinate system of the mapper. All components of the probe's sensitivity tensor are determined by a procedure similar to the one proposed in [4], as illustrated below.

Later, while mapping a magnet, the instantaneous values of the Hall voltages $\vec{V} h$ are measured; and the measured magnetic field vector is calculated, with reference to Equation (1), as

$$
\vec{B}=[S]^{-1} \bullet \vec{V}_{h}
$$

In this way, the effective angular errors of the probe are reduced to below $0.1^{\circ}$, and so the requirement $(d)$ is met.

During the Calibration Algorithm, by measuring the relevant components of the magnetic field, the 9 components of the probe's sensitivity tensor can be determined from the equation

$$
V_{i}=S_{i j} \bullet B_{j}
$$

\section{In Situ Locating of Probe Magnetic Field Sensitivity Spot}

The Magnetic Field Mapper implements an automatic algorithm for the locating the Magnetic Field Sensitive Spot. During this algorithm the Hall probe (Fig. 4) is navigated close to the current-carrying wires.

a)
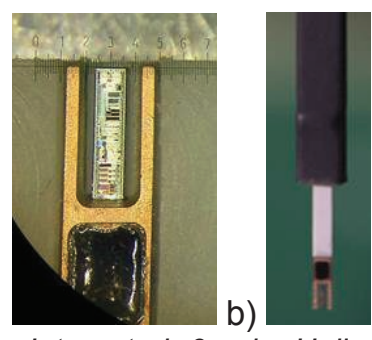

Fig. 4. a) The Integrated 3-axis Hall probe diebonded on a substrate; b) Hall Probe assembled for 
mounting in the $\mathrm{z}$ moving stage of the Magnetic field mapper

In the plane perpendicular to a long current filament parallel with the y-axis (Fig. 5), the magnetic field does not contain the By component; exactly above the wire, there is only $\mathrm{Bx}$ component; and left and right of the wire center, there is only $\mathrm{Bz}$ component. a)

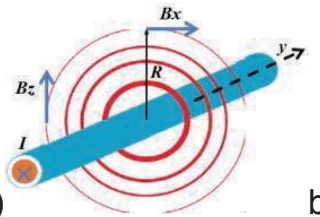

b)

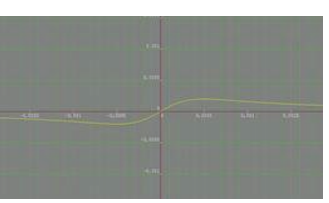

Fig. $5 . \quad$ a) The magnetic field distribution around the conducting wire; b) The Bz magnetic field component as function of distance, measured while navigating the Hall probe close to and over the conducting wire. The Bz component is zero exactly over the wire center.

The calibration tool consists of two PCBs, with the current-conducting Cu-layer. The Hall probe is navigated close to the center of currentcarrying wire that is mounted on the calibration tool (Fig. 6). The probes sensitive volume contains the Hall-elements with their sensitivity vectors. a)

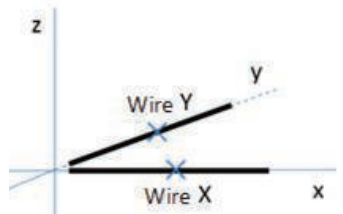

b)

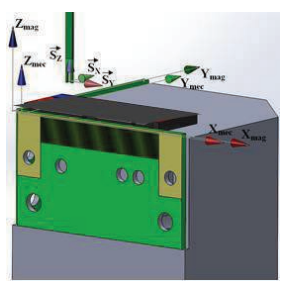

Fig. 6 . a) The magnetic field distribution around the conducting wire; b) Calibration wires $x$ and $y$ in the mapper coordinate system.

The position of the Magnetic Field Sensitive Spot is determined relative to the coordinate system of the mapper with accuracy of $\pm 2 \mu \mathrm{m}$.

Since the magnetic field sensitive volume of the Hall probe is well defined and tightly related to the mapper's coordinate system, the only task to be done is to touch by a stylus the Calibration Tool in $\mathrm{x}, \mathrm{y}$ and $\mathrm{z}$ direction and to store the coordinates obtained. Then the offset between those coordinates and the coordinates obtained during the calibration have to be aligned.

The touch-stylus is used to determine the Mechanical Reference Point MeRP in the mapper's coordinate system. The $X$ any $Y$ PCB's will be touched in three positions by touch-stylus. The position of the Magnetic Field Sensitive Spot cannot be determined in the mapper's coordinate system directly, though it can be determined with regard to the Magnetic Reference Point MaRP (Fig. 7).

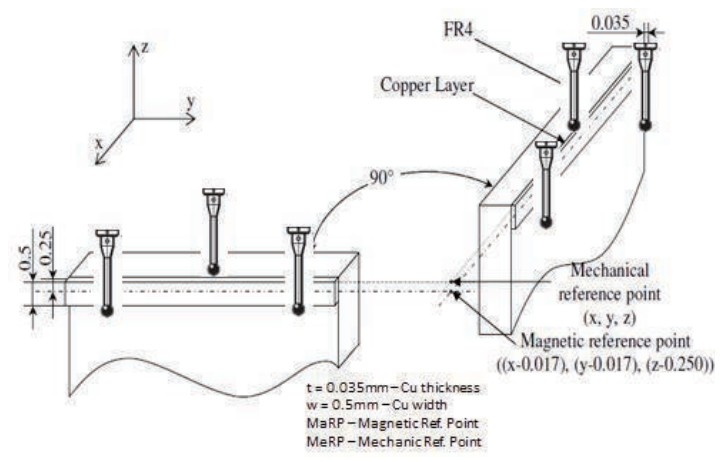

Fig. 7. Process of defining the relation between the magnetic field sensitive spot of the Hall probe and the mapper's coordinate system

The relation between the two reference points is given by equation, which is based on the fundamentals of physics - the center of the infinite current-warring wire is the center of the magnetic field lines, generated around the wire. The Cu-layer thickness $(0.35 \mathrm{~mm})$ and $\mathrm{Cu}$-layer width $(0.5 \mathrm{~mm})$ are used to calculate the offsets.

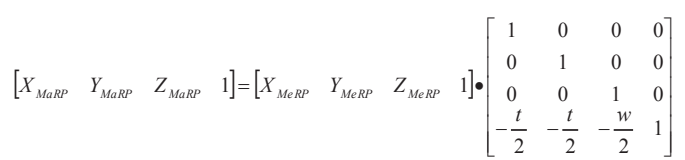

In this way, the direct relation between the magnetic field sensitive spot of the Hall probe and the mapper's coordinate system can be established.

\section{Calibration of Probe Sensitivity Tensor}

The Calibration of Probe Sensitivity Tensor is performed by a reference magnet, integrated in the calibration cube tool (Fig. 8). The reference magnet generates a very homogeneous magnetic field of $90 \mathrm{mT}$ in the volume of $2 \mathrm{~mm} \mathrm{x}$ $2 \mathrm{~mm} \times 2 \mathrm{~mm}$.

The Calibration Cube is characterized in the way that the vector of the magnetic field within the cube is very accurately defined with respect to the cube edges, i.e. to the cube coordinate system.

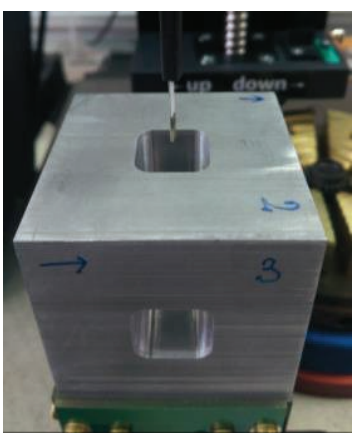

Fig. 8. Tool for the Calibration of Probe Sensitivity Tensor 
The Hall probe is positioned in the center of the homogeneous area of the reference magnet within the Calibration Cube (Fig. 9). During calibration process the Calibration Cube with the reference magnet is rotated around $x, y$ and $z$ axis. Even if the probe is not perfectly orthogonal its magnetic field sensitive spot remains in the homogeneous area of the reference magnet while rotating the Calibration Cube.

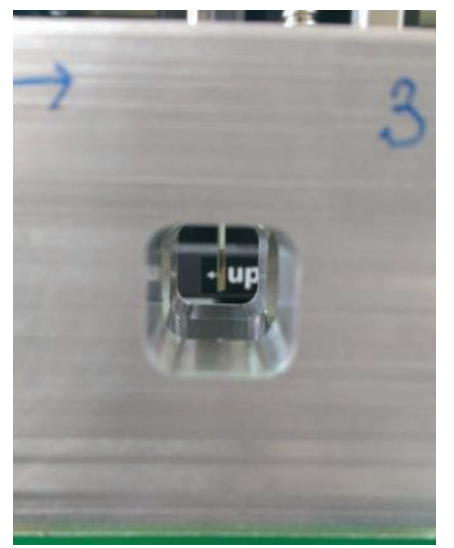

Fig. 9. The Hall probe positioned in the center of the Calibration Cube.

The calibration process requires that the magnetic field vector in the Calibration Cube is always well defined with respect to the coordinate system of the Magnetic Field Mapper. This was achieved by Calibration Cube characterization.

During the calibration process all three components of the magnetic field of the reference magnet will be measured in all different positions of the Calibration Cube.

\section{Experimental Results}

In order to verify the Calibration algorithm, the Hall probe was deliberately twisted, so that a high orthogonality error was produced. The sensitivity of each Hall element integrated in the Hall probe is $100 \mathrm{~V} / \mathrm{T}$ (Sxx, Syy, Szz).

After the measurement of the magnetic field with the Hall probe the following Sensitivity Matrix was obtained:

$$
[S]=\left[\begin{array}{lll}
S x x & S x y & S x z \\
S y x & S y y & S y z \\
S z x & S z y & S z z
\end{array}\right]=\left[\begin{array}{ccc}
96.6577 & 25.6285 & 2.44729 \\
-25.5293 & 96.6622 & 1.95327 \\
-2.4162 & -2.69085 & 99.9925
\end{array}\right]
$$

where all numerical values in the Sensitivity Matrix are in $\mathrm{V} / \mathrm{T}$.

The inverse matrix is:

$[S]^{-1}=\left[\begin{array}{ccc}0.00966308 & -0.00256721 & -0.000186253 \\ 0.002546 & 0.00966328 & -0.000251076 \\ 0.000302011 & 0.00019801 & 0.00998949\end{array}\right]$
After completed Calibration of Probe Sensitivity Tensor, the same measurement of the magnetic field was performed, without moving the Hall Probe. The Hall probe is still twisted in the Calibration Cube, so that this repeated measurement shall provide a much improved sensitivity matrix, which would validate the Calibration Algorithm.

The following Sensitivity Matrix is now obtained:

$[S]=\left[\begin{array}{lll}S x x & S x y & S x z \\ S y x & S y y & S y z \\ S z x & S z y & S z z\end{array}\right]=\left[\begin{array}{ccc}100.002 & 0.0298746 & -0.0089158 z \\ -0.103873 & 100.038 & 0.0110116 \\ 0.0138412 & -0.0328856 & 100.041\end{array}\right]$

The matrix (7) can be re-written in the form

$$
[S] \approx S S \bullet[I]
$$

where $S_{\mathrm{s}}=100 \mathrm{~V} / \mathrm{T}$ denotes the common magnitude of all three sensitivity vectors of the probe, and [I] denotes the unity matrix. The fact that the calibration transforms a highly nonregular sensitivity tensor of the probe (5) into an almost ideal form (8) [4] demonstrates a high efficiency of our calibration procedure.

\section{Conclusion}

The Magnetic Field Mapper with an integrated 3-axis Hall probe allows a very accurate measurement of all three components of the magnetic field in a single point. The orthogonality error of the Hall elements integrated in the single Hall probe Silicon chip and of the Hall probe mounted in the Mapper moving platform can be significantly reduced by the novel calibration algorithm. This unique feature, Calibration of Probe Sensitivity Tensor, enables a user to check and/or recalibrate the 3 -axis Hall probe in-situ, without removing the probe from the mapper. The calibration includes the measurement of magnitudes and signs of all three components of each of the three sensitivity vectors of the three-axis Hall probe (that are treated as a second-order sensitivity tensor or a $3 \times 3$ matrix). The accuracy of the sensitivity magnitude is $+/-0.1 \%$ or $50 \mu \mathrm{T}$.

The embedded calibration software allows a precise angular alignment of the three effective sensitivity vectors with the Cartesian coordinate system of the mapper to within $+/-0.1^{\circ}$. Such an alignment is crucially important in high-accuracy mapping of magnetic field vectors.

\section{References}

[1] M. Blagojevic, N. Markovic, R. S. Popovic, Testing the Homogeneity of Magnets for Rotary Position Sensors, INTERMAG IEEE International Magnetics Conference May 48, 2014, Dresden, Germany 
[2] T. Schliesch, Two Pole Injection Molded Magnets for Measuring Rotating Angles, Magnetics 2015 Conference, Jan. 21-22, 2015, Orlando FL, USA

[3] S. Spasic, I.J. Walker, M. Blagojevic, N. Markovic, Integrating Magnetic Field

Mapping and Coordinate Measurement, Magnetics 2015 Conference, Jan. 21-22, 2015, Orlando FL, USA

[4] D. Popovic Renella, S. Dimitrijevic, S. Spasic, R.S. Popovic, High-Accuracy Teslameter with Thin Three-Axis Hall Probe, IMEKO September 15-17, 2014, Benevento, Italy

[5] D. Popovic Renella and S. Spasic, HighAccuracy 3-axis Teslameter and its application in the 3D Mapping of Magnetic Fields, UK Magnetic Society International Event on Magnetic Materials and Applications, Oct. 28-29, 2014, Lucerne, Switzerland

[6] D. Popovic Renella, S. Dimitrijevic, M. Blagojevic, P. Kejik, E. Schurig, R.S. Popovic, Three-Axis Teslameter With Integrated Hall Probe, IEEE Transactions on Instrumentation and Measurement, vol. 56, No. 4, August 2007

[7] H. Husstedt, Measurement of Magnetic Fields for the Testing of Automotive Sensors, Dissertation March, 2014, AlpsAdriatic University Klagenfurt, Austria 